\title{
Por una estética de la naturaleza: la belleza natural como argumento ecologista
}

\author{
MARTA TAFALLA \\ Universidad Autónoma de Barcelona
}

RESUMEN. En este artículo defiendo la tesis de que la estética de la naturaleza puede ofrecer a la ética de la naturaleza uno de los mejores argumentos ecologistas. Creo que el valor estético de la naturaleza, tradicionalmente olvidado por la filosofía, puede revitalizar las discusiones que tienen lugar en la ética de la naturaleza, y permite enfocar de un modo nuevo la cuestión de si la naturaleza posee un valor intrínseco o un valor instrumental.

\section{LA EXPANSIÓN DEL CÍRCULO DE LA MORAL}

Aunque ya no cabe la menor duda de que la catástrofe ecológica que estamos provocando, y que no nos hace desmerecedores de la controvertida metáfora del cáncer, se ha convertido en la mayor crisis que ha tenido que afrontar la humanidad a lo largo de su historia y, a pesar de que no existe tarea más urgente que encontrar soluciones prácticas para cada uno de los diversos factores implicados en la drástica pérdida de biodiversidad o en la contaminación y el consecuente calentamiento del planeta, sin embargo, todavía somos inca-
AbstRact. In this paper, I defend the thesis that aesthetics of nature can offer to the ethics of nature one of the best ecological arguments. I believe that the aesthetic value of nature, which was traditionally neglected by philosophy, can revitalize the discussions which take place in the ethics of nature, and allows to approach in a new way the question if nature has an intrinsic or an instrumental value. 
conducen inevitablemente a algunas de las más decisivas y difíciles preguntas filosóficas, a cuestionarnos la naturaleza y el alcance de la moral, a definir cuál es el lugar del ser humano dentro del reino de la vida, o a decidir, a fin de cuentas, cómo queremos vivir y convivir en este planeta.

Extender el círculo de la moral más allá de la especie humana parece ahora una necesidad, y, sin embargo, ¿hasta dónde debemos ampliarlo? Si examinamos brevemente el proceso por el cual la moral intenta trascender la especie humana, podemos distinguir en él dos fases diferenciadas. En la primera, que resulta todavía relativamente sencilla en comparación con la segunda, la moral se extiende para abrazar a los animales, y en especial, a aquellos a los que los seres humanos hemos integrado en nuestra forma de vida, ya sea porque los hemos adoptado como animales de compañía, o bien porque los utilizamos como instrumentos en la producción de alimentos, la experimentación o el entretenimiento. En este caso, extender el círculo de la moral es una consecuencia razonable de una convivencia secular. $Y$ los intentos de esa extensión son por ello igualmente seculares. Si el budismo ofreció en Oriente una moral de compasión hacia los animales, en Occidente esta cuestión se remonta a diversos filósofos griegos y romanos que fueron ya defensores del vegetarianismo, como Pitágoras de Samos, Plutarco de Queronea o Porfirio de Tiro; y de Plutarco sabemos también que se opuso públicamente a los cruentos espectáculos con animales del circo romano. $Y$ aunque el cristianismo predominante en la filosofía medieval silenció esas voces, sus demandas no tardaron en reaparecer en las voces de la Ilustración. Autores como Hume, Bentham, Rousseau, Voltaire, Leibniz o Kant, no sólo coincidieron en su defensa de las libertades humanas y su lucha por sociedades más justas, sino también en reclamar un trato más humano para con los animales.
Lo que permite realizar esa primera fase de la expansión sin demasiados problemas teóricos, es que no necesitamos cambiar de lenguaje; la mayoría de conceptos básicos con los que hemos construido teorías morales para los seres humanos, pueden seguir siendo utilizados cuando extendemos la moral a nuestra relación con los animales. Esto es así porque, en primer lugar, los animales son seres individuales. Ese chimpancé que hemos visto en el zoo, el perro que encontramos extraviado en la carretera, aquel elefante que actúa en el circo, la ballena varada en la playa, el cerdo que muere en el matadero, son seres a los que podemos dar un nombre (y algunos responden a él), que tienen una historia que podría ser narrada ( $y$ en algunos casos así se ha hecho), que poseen personalidad, sentimientos e inteligencia, aunque en diferente grado, y con los que podemos relacionarnos. $\mathrm{Y}$ en segundo lugar, porque son seres a los que podemos causar sufrimiento físico y psíquico, y en cuya conducta podemos reconocer ese sufrimiento; por lo que el dolor en sus diversos tipos funciona como un indicador básico (aunque no siempre necesario ni suficiente) para detectar cuándo esas criaturas son tratadas de forma injusta. Así pues, emplear nuestro vocabulario moral tradicional y hablar de esclavitud, tortura, crueldad, maltrato, para referirnos a los animales, tiene pleno sentido. Hasta el punto de que, según diversos teóricos, la expansión del círculo de la moral a los animales puede entenderse como la continuación de un proceso histórico por el que la humanidad ha ido superando, una tras otra, las ilusorias fronteras del racismo, el sexismo y finalmente el especismo.

Esta ampliación también puede explicarse en otros términos: en los de expansión de la moral más allá de las relaciones recíprocas. Desde hace unas décadas son muchos y de diversas procedencias teóricas los pensadores que trabajan en territorios de la moral antes considerados limí- 
trofes o marginales debido a esta ausencia de reciprocidad. Determinar qué deberes morales tenemos hacia aquellos seres humanos que no pueden reconocer ni asumir deberes hacia nosotros, ya sea porque son enfermos en estado vegetativo, o porque padecen graves carencias psíquicas, nos obliga a formular teorías morales que no pueden basarse en contratos ni diálogos, en los que no podemos confiar en que los otros nos traten como los tratemos, y ni siquiera esperar el mero reconocimiento si los tratamos con justicia. Aún más, los movimientos que reclaman justicia para víctimas que ya han fallecido, o que reivindican los derechos de las generaciones futuras, extienden la moral no sólo más allá de la reciprocidad, sustituyendo el diálogo por la memoria o por la imaginación, sino incluso, en el segundo caso, para exigirnos deberes morales hacia seres que ni siquiera sabemos si existirán. Nuestra relación con los animales es sólo uno más de los diversos ámbitos en los que la moral se expande trascendiendo la reciprocidad. $Y$ en todos estos casos, sabemos que existe una sencilla razón por la cual la moral no puede detenerse allí donde la reciprocidad deja de ser posible: porque aquellos seres (humanos o no, vivos o no) que no son capaces de comportarse moralmente, que no son propiamente agentes morales, son aquellos que más fácilmente acaban siendo víctimas de injusticias. Simplemente porque el no ser agentes morales no sólo significa que no pueden comportarse con justicia hacia los demás; significa asimismo que no pueden exigirla para sí, que no son capaces de defenderse o denunciar cuando son maltratados.

De todos modos, si alguien sigue considerando que los animales no merecen consideración moral porque ellos mismos no son agentes morales con los que podamos dialogar o firmar contratos, es conveniente prestar atención al argumento que se está empleando. Si decimos que, porque nosotros somos agentes morales y los otros animales no lo son, entonces podemos maltratarlos impunemente, resulta que hemos convertido el hecho de ser agentes morales en una justificación para maltratar. ¿Dónde queda entonces la moralidad del agente moral?

Por todas estas razones, argumentar a favor de un trato justo hacia los animales es una cuestión relativamente sencilla, y la mayoría de teóricos se limitan a extender a las otras especies animales las mismas teorías morales que hemos elaborado para la nuestra. Así, existen defensores de los animales que son utilitaristas, otros que emplean una moral de la compasión de raíz schopenhaueriana, los que buscan argumentos en Kant, o los que toman la idea de los derechos humanos y tratan de aplicarla como derechos de los animales ${ }^{1}$. Y subrayo esa relativa sencillez por contraste, porque una vez intentamos avanzar un paso más, e iniciamos la segunda fase de la expansión del círculo de la moral, no sólo más allá de la especie humana, sino también del reino animal, dejamos atrás toda posibilidad de extender nuestras teorías morales tradicionales.

Cuando comenzamos a preocuparnos por la naturaleza en su conjunto, y analizamos problemas tales como la contaminación del ártico o la destrucción de los fondos marinos, con el fin de intentar esclarecer cuáles son nuestras obligaciones morales al respecto, nuestro lenguaje moral tradicional comienza a fallarnos. $Y$ nos falla, en primer lugar, porque esos problemas ya no pueden plantearse al nivel de seres individuales. Lo relevante aquí son los sujetos colectivos: por un lado, las especies, y por otro, los ecosistemas. $\mathrm{O}$ incluso hay quien argumenta que el verdadero sujeto que merece consideración moral es la biosfera, puesto que todas las formas de vida conforman una unidad que es inútil e ilusorio dividir en unidades menores, ya que los problemas de una especie o de un ecosistema tienden a acabar afectando a otros. $Y$ en segundo lugar, 
porque si los conceptos de mal o de injusticia todavía siguen siendo válidos para referirnos a las especies, los ecosistemas o la biosfera, entonces ya nada tienen que ver con el dolor, sino con la pérdida de biodiversidad o con el desequilibrio.

Y la complejidad no acaba aquí. Encontrar buenas razones para proteger la naturaleza también incluye dar buenas razones para preservar elementos naturales que ni siquiera están vivos. Esas razones también deben ser capaces de explicar por qué no deberíamos destruir las estalactitas y estalagmitas que han tardado miles de años en formarse en una gruta, o por qué no deberíamos destruir las rocas modeladas por el viento de la ciudad encantada de Cuenca para construir bloques de apartamentos en su lugar. Destruirlas nos parece un mal, pero ¿qué tipo de mal es ése? ¿Puede una entidad inorgánica ser víctima de un mal? ¿Podemos ser injustos con una piedra? ¿Por qué sería incorrecto convertir la luna en un vertedero, si ni está viva ni la habita ningún ser vivo?

Y no olvidemos, para añadir una última dosis de complejidad, que aunque asumamos algún tipo de deber moral hacia la naturaleza, renunciamos de entrada y por razones obvias a introducir la moral en el ámbito de las relaciones entre el resto de seres vivos. Es decir, consideramos que es justo preservar la naturaleza tal y como es, a pesar de que en ella las criaturas vivan sometidas a unas leyes amorales que las obligan a vivir vidas de crueldad y de dolor. Con lo cual asumimos la obligación moral de preservar un reino de la vida amoral y cruel. $Y$ eso otorga un carácter trágico a cualquier ética de la naturaleza, como ya nos enseñaron Schopenhauer o Darwin, o como en el fondo nos han enseñado desde hace siglos el budismo y el judaísmo. Debemos tratar con justicia a una naturaleza que no será jamás un reino justo para sus habitantes, por mucho que el Génesis se inicie con la añoranza de un primigenio jardín del Edén donde la natu- raleza no incluía la violencia y los animales no se devoraban entre sí, o que Isaías predijera una naturaleza reconciliada en cuyo seno el león pacerá con el cordero.

Dada toda esta serie de dificultades, ¿qué buenas razones podemos encontrar para proteger la naturaleza?

\section{EN BUSCA DE BUENAS RAZONES PARA PROTEGER LA NATURALEZA}

El debate filosófico en busca de esas razones se ha dividido en dos grandes corrientes opuestas, que se subdividen a su vez en muchas otras ${ }^{2}$. Sintetizado de manera brevísima, la posición más sencilla y más frecuente nos dice que la mejor razón para proteger a la naturaleza somos nosotros, mientras que la posición más minoritaria y difícil argumenta que la mejor razón para proteger a la naturaleza radica en ella misma.

La primera de estas posiciones concibe el valor de la naturaleza como un valor instrumental para un sujeto. La versión mayoritaria de esta posición afirma que ese sujeto somos nosotros mismos (lo cual puede incluir a las generaciones futuras), y se denomina por ello antropocentrismo. En una versión minoritaria, el sujeto son todos los animales entendidos individualmente, y hablamos de zoocentrismo. En ambos casos la naturaleza es concebida como el hábitat, fuente de recursos, materiales, energías u objeto de estudio, y la razón para protegerla es, por tanto, una razón egoísta.

Los detractores de esta posición han intentado superar sus limitaciones afirmando que la naturaleza, lejos de reducirse a un mero instrumento, posee un valor intrínseco, y es en realidad un fin en sí misma. Esta posición, denominada biocentrismo, no sólo expande el círculo de la moral hasta abrazar toda la naturaleza, sino que expulsa al ser humano de su centro y entrona en su lugar a la vida misma, 
con lo cual podría entenderse como el último y definitivo paso en el proceso de descentramiento del ser humano en el orden de la realidad que se inició con Galileo y prosiguió con Darwin. Algunos de sus defensores denuncian la posición anterior como ecología superficial y apuestan por el salto a una ecología profunda; y aunque hay algo de cierto en intentar superar así la egoísta perspectiva humana, más cierto es aún que en la prometida profundidad de las teorías biocentristas nos acechan peligros nada desdeñables.

En primer lugar, lo que se hace evidente con celeridad es que instaurar la vida como centro del círculo de la moral significa en realidad disolver la moral y sumirse en la amoralidad natural. Las teorías biocentristas identifican la vida o lo natural con lo bueno, $y$, en consecuencia, se precipitan en la falacia naturalista. Además, algunas de ellas extraen la conclusión de que ciertas formas de violencia pueden ser justas, siempre que sean naturales (por ejemplo, la caza, y aquí hallamos uno de los puntos de enfrentamiento entre ecologistas biocentristas y defensores de los animales). Y en segundo lugar, una amplia mayoría de biocentristas se declaran holistas, disolviendo al individuo en la especie, en el ecosistema o simplemente en el todo de la naturaleza. Me parece obvio que conceder mayor peso a entidades supraindividuales y amorales como los ecosistemas o la biosfera que a los seres humanos, es una vía segura para acabar perdiendo de nuevo los derechos y libertades que tanto tardamos en lograr. (Si ponemos los intereses de la biosfera absolutamente por encima de los nuestros, el mayor interés de ésta es, sin duda alguna, que reduzcamos de inmediato nuestra población en un 90 por 100.) En realidad, el biocentrismo no es exactamente una teoría moral. Tiende a suspender la moral por renunciar a la perspectiva individual y por sumisión a la amoralidad de la naturaleza, y se construye como una cosmovi- sión metafísica, religiosa o mística, algunas de cuyas versiones, demasiado inspiradas en Heidegger, se sitúan más bien a la ultraderecha. Como advierte Tom Regan, en el holismo acecha el totalitarismo ${ }^{3}$.

Así pues, si todo cuanto podemos hacer es escoger entre el egoísmo de fondo del antropocentrismo, que es un límite para formular razones sólidas de protección de la naturaleza, y la suspensión de la moral en el biocentrismo, más que ante dos opciones, parece que nos encontremos ante el cruce de dos callejones sin salida.

Tras afirmar la imposibilidad de encontrar razones morales para proteger la naturaleza, y describir las dificultades para formular razones éticas, concluye Jürgen Habermas: «En algunos aspectos, las razones estéticas son incluso de más peso que las éticas. Pues en la experiencia estética de la naturaleza, las cosas se retiran por así decir a una inaccesible autonomía e intangibilidad, y sacan entonces a la luz su vulnerable integridad con tanta claridad que nos parecen inviolables por sí mismas, y no meramente como partes deseadas de una forma de vida preferida» ${ }^{4}$. ¿Es esto tan sólo la excusa de un filósofo moral intentando pasarle a otra disciplina un problema que él no sabe resolver? ¿O deberíamos tomarnos la idea en serio? J. Baird Callicott, un biocentrista de cuya preocupación ecologista no cabe duda, reconoce igualmente: «many more of our conservation and preservation decisions have been motivated by beauty than by duty ${ }^{5} \gg . \iota^{\mathrm{PO}-}$ dría ése ser realmente un camino? ¿Podría la estética abrimos un camino nuevo allí donde la colisión entre antropocentrismo y biocentrismo nos dejan atrapados?

\section{LA BELLEZA NATURAL COMO ARGUMENTO}

Suspendamos por ahora la discusión sobre si la naturaleza posee un valor intrínseco o instrumental. De lo que no nos cabe duda, 
es que posee un valor estético, pues está llena de seres vivos, entidades inorgánicas y paisajes que reconocemos como bellos. $\mathrm{Y}$ que reconocemos esa belleza significa que nos deleitamos en su contemplación: que nos agrada contemplar flores de hibisco de las que vienen a alimentarse los colibríes; que pasear por una playa desierta en una mañana de invierno nos devuelve la calma perdida en la ciudad; que contemplar altas cumbres nevadas nos despierta sentimientos de admiración, humildad y respeto. La belleza natural, en su infinidad de formas, incluso es capaz de provocarnos una sensación de libertad, de encontrarnos con nosotros mismos; por ello a menudo escogemos parajes naturales para retirarnos por unas horas a pensar, a tomar decisiones o a recuperarnos de un disgusto. $Y$ más allá de lo que percibimos de forma inmediata, si un microscopio nos invita a penetrar en lo minúsculo, o un telescopio nos permite viajar con la mirada a lo más lejano, lo que descubrimos continúa siendo bello. (De algunas de esas cosas diríamos incluso que, más que bellas, son sublimes, pero dada la brevedad de mi exposición, no puedo abordar aquí la cuestión de lo sublime natural.) Creo que la mayoría de seres humanos valoramos esas experiencias, y que vernos privados de ellas nos parecería un mal. Así pues, ¿podría la belleza de la naturaleza alzarse como una buena razón para protegerla?

En una primera impresión, podría parecer que mi argumento «debemos proteger la naturaleza porque está llena de cosas bellas» en el fondo es equivalente a «debemos proteger la naturaleza porque a los seres humanos nos produce placer» $\mathrm{y}$, por tanto, sería un argumento del mismo tipo que «debemos proteger la naturaleza porque está llena de productos que podrían usarse en la industria farmacéutica». Y, sin embargo, ¿es el valor estético un valor instrumental? Considerar que la naturaleza tiene un valor estético, ¿implica reducirla a un mero instrumento?
Cuando yo digo que la música de Mahler, la pintura de Pollock o la literatura de Marsé me proporcionan experiencias estéticas muy placenteras, nadie me responde que estoy instrumentalizando esas obras de arte. En realidad, estoy haciendo todo lo contrario. La mirada estética interrumpe el quehacer práctico, pone fin al reino de la razón instrumental y a nuestras ansias de dominio más egoístas sobre todo cuanto existe. Por ello, en cuanto le reconocemos a un objeto un valor estético, dejamos de verlo como un instrumento, dejamos de usarlo como un medio para conseguir nuestros fines, y en vez de eso, nos detenemos para contemplarlo y admirarlo. Nuestra voluntad se detiene ante él. Lo respetamos. Ésa es una intuición que los filósofos del arte llevan siglos intentando conceptualizar, e incluso podría afirmarse que la historia de la estética ha sido la historia de intentar diferenciar la esfera de lo estético de la esfera de la utilidad. Kant lo formuló con la idea del desinterés. Schopenhauer argumentó que la contemplación estética era capaz de suspender la ansiedad permanente de la voluntad de vivir en su lucha por la supervivencia. Adorno buscaba en la experiencia estética el fin de la voluntad de dominio sobre todo cuanto existe, y el lugar donde el ser humano puede descubrir y aprender a valorar lo diferente de sí mismo.

Es cierto que el arte también nos permite realizar, además de experiencias estéticas, experiencias de aprendizaje moral, de protesta política o de muchos otros tipos. Pero lo importante aquí es que esa experiencia estética es posible. Así pues, si la experiencia estética del arte nos permite tener con él una relación no instrumental, la experiencia estética de la belleza natural también debe permitirnos tener una relación no instrumental con la naturaleza. Mientras que normalmente tendemos a concebir y valorar la naturaleza como una fuente de materias primas, energía, alimentos, productos farmacéuticos 0 
como un objeto de estudio, la experiencia estética nos abre a otra forma de verla y valorarla. Somos capaces de extraerla de la esfera de la utilidad y contemplarla tal como lo hacemos con las obras de arte. En esos momentos se detiene nuestra voluntad de dominio, nuestra razón instrumental y los cálculos de utilidad, y nos limitamos a admirarla y respetarla. Y es así como la experiencia estética de la belleza natural nos enseña a proteger la naturaleza. Aunque la naturaleza sigue siendo una fuente de recursos que necesitamos para vivir, aprender a admirarla estéticamente nos enseñará a limitarnos, a poner límites en nuestro uso de la naturaleza.

$\mathrm{Y}$ aquí recuperamos un hilo argumental anterior. Habíamos dicho que la mayor dificultad para hablar de la protección de la naturaleza es que nos falla el lenguaje, que los conceptos morales de siempre ya no nos sirven. ¿Podría ser entonces que el lenguaje más apropiado para hablar de la protección de la naturaleza fuese en realidad el mismo lenguaje que usamos para hablar de la conservación de las obras de arte?

Cuando la mala gestión de la catástrofe del Prestige inundó de chapapote las playas gallegas, afirmamos con indignación que eso era un mal, y exigimos un plan urgente de recuperación de las zonas afectadas. ¿No es ésa la misma indignación que sentimos al saber que los soldados estadounidenses en Irak están dañando gravemente las ruinas de la ciudad de Babilonia y exigimos que sean restauradas? ¿No es el mismo tipo de mal? La desaparición de culturas minoritarias, la pérdida de diversidad cultural, ¿no es una pérdida del mismo tipo que la extinción de especies, que la disminución de biodiversidad? Nuestro deseo de que no desaparezcan culturas, géneros artísticos, tradiciones artísticas, ¿no es afín a nuestro deseo de que no se pierdan ecosistemas o especies? ¿No existe además una profunda semejanza entre una cultura y un ecosistema, en tanto que entidades supraindividuales que son algo más que los individuos que las forman? ¿No coinciden incluso nuestras dificultades para hablar moralmente de ambas, y el peligro de ponerlas demasiado por encima de los derechos de los individuos? $\mathrm{O}$, por otra parte, la ambigüedad que sentimos ante los museos, con nuestra admiración hacia su voluntad de preservar elementos de culturas ya desaparecidas, pero al mismo tiempo la añoranza hacia esas culturas cuyos restos sólo citan y evocan, o a menudo la indignación porque esos museos son en realidad botines de guerra de los vencedores, que a veces incluso traicionan el sentido de la cultura expuesta, ¿no recuerda la ambigüedad de los zoos, al mismo tiempo ecos del intento de Noé de salvaguardar la diversidad de la vida, pero que a menudo parecen más bien la exposición del botín de una guerra ganada contra la vida salvaje?

Mi tesis es que para elaborar un lenguaje con el que defender la preservación de la naturaleza, podemos aprender más del lenguaje sobre la conservación del arte y la cultura, que no del lenguaje moral tradicional. Y creo que eso no sólo es válido para las semejanzas, que acabo de intentar sugerir, sino también para las profundas diferencias que existen entre la experiencia estética del arte y la experiencia estética de la naturaleza. Veamos ahora esas diferencias.

La primera gran diferencia tiene que ver con los sentimientos y pensamientos sobre nosotros mismos que las experiencias estéticas nos provocan. Cuando admiramos la pintura de Pollock, esa admiración nos conduce de manera casi inmediata a admirar a su autor, de ahí a admirar la cultura de la cual surgió su obra, y finalmente, a enorgullecernos de la inteligencia y la creatividad del ser humano. Delante de uno de los cuadros de Pollock, podemos decirnos que a pesar de todo lo que hacemos mal, la especie humana merece la pena aunque sólo sea 
por las obras que ha creado, por haber inventado la pintura, la música o la literatura, y haber hecho el universo más hermoso.

Ahora bien, cuando es la naturaleza quien nos cautiva, cuando nos maravillan las formas, los colores y los cantos de las aves o cuando reconocemos la magnificencia del firmamento estrellado, reconocemos al mismo tiempo todo lo que no somos capaces de crear, la belleza que ya existía en el universo antes de nuestra llegada y que en cambio nosotros estamos poniendo en peligro. Gozar de la belleza natural es también una lección de humildad, que nos invita a tomar conciencia de nuestra pequeñez frente a las maravillas de la naturaleza, ya sean criaturas vivas, lunas, estrellas o nebulosas. Esa belleza nos recuerda nuestras limitaciones, pero también nos enriquece al permitirnos ver más allá de nosotros mismos. Asumir la propia finitud significa abrirse a descubrir criaturas y paisajes que no seríamos capaces de inventar. Incluso cuando intentamos colaborar con la naturaleza, cuando practicamos el arte milenario de la jardinería, o su versión actual de la gestión de paisaje, sabemos que no somos verdaderos artistas, sino que nos parecemos más bien al curador de una exposición o el conservador de un museo, que cuida, reordena y presenta aquellas obras que no es capaz de crear.

Existe una segunda diferencia que resulta incluso inquietante. En las obras de arte, que son obra nuestra, no podemos entrar. Las obras de arte son la invención de otros mundos, pero esos mundos rehúsan nuestra entrada en ellos. Por mucho que las obras existan físicamente, están definidas y protegidas por unos marcos que no podemos traspasar; no son en realidad más que mundos cerrados en sí mismos. Una pintura podemos contemplarla, una novela leerla, una sonata escucharla, pero siempre existe una distancia infranqueable entre la obra y nosotros. Tan sólo la arquitectura nos permite entrar en ella, pero por ello mismo siempre ha sido considerada la forma artística donde el arte se confunde en mayor grado con la utilidad.

En cambio, la naturaleza, que no es obra nuestra, carece de límites y marcos, y no sólo nos lo permite, sino que nos invita a entrar en ella y recorrerla. La experiencia estética de la naturaleza es la experiencia de algo que se abre para acogernos, que nos envuelve. Dejamos de ser meros espectadores distantes para encontrarnos dentro de ella, participando de ella, descubriéndonos como habitantes, como miembros del mundo natural. Un paseante recorriendo un paisaje pasa a formar parte de ese paisaje, y por eso la naturaleza es la única obra de arte de la que podemos formar parte como individuos físicos, en nuestra corporalidad, si se me permite decirlo así.

La tercera diferencia la encontramos cuando decidimos entrar en la naturaleza. En cuanto nos adentramos en un bosque o emprendemos el ascenso a una montaña, la naturaleza nos ofrece estímulos para los cinco sentidos; en ella podemos acariciar la corteza de los árboles, oler la hierba mojada tras la lluvia, escuchar cantar a los mirlos, probar si las moras ya están maduras, y contemplar la luz de la mañana. Y no sólo apela a nuestros cinco sentidos, sino a todas nuestras capacidades. Tanto puede despertarnos la atención y la curiosidad del naturalista que observa una oruga en la palma de su mano, como estimular nuestra imaginación o los más diversos sentimientos. Y ese mundo que nos habla en todas las lenguas que nuestro cuerpo reconoce, no cesa de hablarnos nunca, porque es un mundo vivo, plural y en constante transformación, impredecible e inagotable regalador de sorpresas. No hay dos instantes idénticos, nada vuelve a repetirse jamás. Aquella noche de agosto en que fuimos a la playa a contemplar las estrellas fugaces es irrepetible, como lo es la nevada que nos sorprendió de excursión 
en la montaña. Nunca vuelve a darse la misma combinación de luz, colores, olores, sonidos y sabores. Uno vuelve una y otra vez al mismo lugar preferido, y cada vez es distinto. La experiencia estética de la naturaleza es de una riqueza, pluralidad y diversidad infinitas, capaz de proporcionarnos formas distintas de belleza por toda la eternidad. Si este tipo de experiencia desapareciera, no podríamos sustituirla por ninguna otra. El arte y la naturaleza nos provocan algunas de las experiencias más placenteras que podemos tener a lo largo de nuestra vida, pero ninguno de los dos puede sustituir al otro. Si perdiéramos la belleza de la naturaleza, si perdiéramos la diversidad de especies y de paisajes, ninguna obra de arte podría compensarnos por esa pérdida. La belleza natural, una vez destruida, no puede ser recuperada. Y es así, por tanto, mucho más frágil que nuestras obras de arte, de las que siempre podemos al menos hacer copias.

Así caracterizada brevemente la experiencia estética de la naturaleza ( $y$ soy consciente de que tal brevedad deja algunos cabos sueltos que aquí no tengo espacio para abordar), creo poder afirmar que su valor estético es una razón fuerte y sólida para proteger la naturaleza. ¿Dónde se sitúa este argumento en la discusión entre antropocentristas y biocentristas? Tal como he afirmado, el valor estético no es un valor instrumental, sino que, todo lo contrario, nos enseña a valorar la naturaleza como algo que no es un mero instrumento. Sin embargo, tampoco podemos asumir que el valor estético sea un valor intrínseco del tipo que defienden los biocentristas, pues la experiencia estética es siempre la experiencia de un sujeto que contempla un objeto, y en este sentido toda experiencia estética es antropocéntrica. (Quizás un día descubriremos que algunos animales también tienen experiencias estéticas y hablaremos de zoocentrismo, pero desde luego carece de sentido hablar de biocentrismo de la estética.) $\mathrm{Mi}$ convicción es que el reconocimiento de la belleza natural nos permite debilitar el antropocentrismo, pero evitando precipitarnos en el biocentrismo. Si el peor defecto del antropocentrismo era justamente su egoísmo, creo que éste puede corregirse sin necesidad de disolver al individuo en el todo, que es la drástica solución biocentrista. Creo que el mejor modo de no renunciar a la individualidad ni a la moral, pero que al mismo tiempo nos hace cobrar conciencia de nuestra finitud como seres humanos, es aprender a reconocer y gozar de lo diferente, a descubrir frente a lo diferente nuestros límites y limitar nuestro egoísmo, es simplemente aprender a admirar la belleza natural.

Antes de finalizar, debo hacer dos precisiones respecto al status de este argumento. En primer lugar, el recurso a la estética para lograr un antropocentrismo débil, y dar así una razón fuerte para proteger la naturaleza, no debe verse como una renuncia a la moral. Se trata tan sólo de una de esas muchas y diversas ocasiones en que la estética le echa una mano a la moral. Muchas obras de arte nos han ayudado a plantear con mayor precisión conflictos morales difíciles, han educado nuestra libertad o formado nuestros sentimientos morales. Y aún más, a veces incluso consideramos que algunas obras de arte pueden hacer justicia de algún modo, como cuando una obra recupera la memoria de personas que fueron víctimas de injusticias. Aquí se trata tan sólo de que la mirada estética nos permita ver con mayor claridad dónde están los límites entre el uso del medio natural y su preservación.

La segunda precisión se refiere a que estoy afirmando que el valor estético de la naturaleza es una buena razón para su protección, pero de aquí no debe deducirse que nuestra actividad de conservación de la biodiversidad deba guiarse exclusivamente por un criterio estético. Hacerlo sería un disparate y tendría sin lugar a 
dudas consecuencias catastróficas. No se trata de conservar sólo lo que nos parece más bello y desatender lo que pueda parecernos feo. La belleza natural nos enseña que debemos proteger la naturaleza, pero cómo debamos hacerlo es una pregunta de tipo práctico que debe responderse en cada caso concreto teniendo en cuenta diversas disciplinas científicas.

Aclarados estos aspectos, y asumiendo de nuevo que dejo muchos cabos sueltos en mi exposición, queda, sin embargo, una pregunta central que no puedo obviar. Si la belleza natural es una razón tan buena para proteger la naturaleza, entonces, ¿por qué apenas se la emplea?

\section{EL OLVIDO DE LA ESTÉTICA DE LA NATURALEZA}

A finales de la década de los sesenta del pasado siglo, se alzaron dos voces para protestar por el olvido de la estética de la naturaleza. Una de ellas era la de Ronald Hepburn, quien en 1966 publicó un artículo titulado «Contemporary Aesthetics and the Neglect of Natural Beauty» ${ }^{6}$. La otra era la voz de Theodor W. Adorno en un capítulo de su Teoría Estética, que apareció publicada póstumamente en $1970^{7}$. La relativa coincidencia de fechas no es casual. Ambos, ante la conciencia de una catástrofe que era ya visible, en medio de las primeras voces de alarma, unían a su denuncia de la explotación desmedida de la naturaleza su lamento por el modo como la filosofía había desatendido la estética de la naturaleza. Y no sólo desatendido, sino incluso deslegitimado. Pues aunque en todas las épocas una considerable mayoría de las personas han gozado de un modo u otro de la belleza natural, el hecho de que la filosofía no prestara atención a esas experiencias, les negaba una reflexión teórica que permitiera hablar de ellas con precisión, analizarlas, comprenderlas y defenderlas.
No es que el olvido haya sido unánime, pero sí mayoritario. Cierto es que la estética moderna, que nació en aquella Inglaterra próspera y en paz forjada por la revolución de 1688, en la que florecieron artes y ciencias, nació como reflexión estética sobre el arte y la naturaleza; y que el proyecto kantiano de incluir la estética en su sistema trascendental continuó abrazando a la vez a ambos. Según Adorno, incluso habría que afirmar que las más penetrantes apreciaciones de la Crítica del Juicio son las que se refieren a la belleza natural ${ }^{8}$. Pero esa feliz convivencia apenas se prolongó unas décadas. El idealismo se encargó de ponerle fin, reduciendo la estética a filosofía del arte, y su dictamen fue escrupulosamente respetado por la gran mayoría de filósofos. Así, mientras cada vez más artistas de todas las disciplinas se inspiraban en la belleza natural o la recreaban; y al mismo tiempo que la apreciación estética de la naturaleza estaba presente en muchos viajeros y descubridores; y se convertía en un tema literario desde Melville o Tolstoi a Thomas Mann, la filosofía continuó sin prestarle atención. No fueron filósofos académicos quienes formularon, por ejemplo, lo que denominamos estética positiva de la naturaleza, forjada en los escritos de Henry David Thoreau, John Muir, Aldo Leopold y otros, quienes descubrieron y describieron en el continente americano una naturaleza virgen que ya no existía en Europa, y exaltaron el paisaje no tocado por la mano humana, ofreciendo argumentos que en su día sirvieron para la creación de parques nacionales en Estados Unidos y que hoy siguen siendo de inspiración para muchos ecologistas. Y esa ausencia de reflexión filosófica tiene seguramente mucho que ver con que, cuando Adorno denuncie el olvido de la belleza natural, ya no llegue a tiempo de ofrecer una estética positiva de exaltación de la naturaleza virgen, sino que tenga que limitarse a una estética negativa que descubre a una naturaleza dañada, llena de cicatrices, deforma- 
ciones y ruinas. La belleza natural que a Adorno le toca contemplar es una forma de belleza que reclama ya la ayuda de la memoria, pues ha iniciado el proceso de su desaparición.

¿Por qué ese olvido? Adorno había aprendido de Simmel que la filosofía ha olvidado demasiadas cosas. El dolor, los animales, el paisaje y las mujeres forman la constelación de aquellos temas que han sido persistentemente olvidados por la historia de la racionalidad occidental. Y Adorno nos recordaba que toda forma de olvido es una forma de dominio ${ }^{9}$. Lo que ese olvido encubre es nuestra violencia contra lo olvidado.

Las protestas de Hepburn y Adorno lograron que los filósofos que se ocupaban de la belleza natural dejaran de ser una mera excepción, aunque tan sólo para pasar a ser una digna minoría. Un pequeño grupo de autores en lengua inglesa, como Allen Carlson, Arnold Berleant, Malcolm Budd, Thomas Heyd, Emily Brady, Holmes Rolston III, Noël Carroll y otros, y un reducido grupo de autores en lengua alemana, que incluye a Gernot Böhme y Martin Seel, han emprendido un proceso de rehabilitación de la estética de la naturaleza que pretende devolverla al debate filosófico ${ }^{10}$. Que lo consigan no será independiente de que logremos salvar a la biosfera de la catástrofe que la amenaza.

Los estudios de estos autores, junto con los de algunos biólogos e historiado- res, nos están permitiendo descubrir que el disfrute de la belleza natural siempre ha formado parte de la existencia humana, en todas las épocas y culturas, por mucho que la racionalidad oficial la desatendiera $o$ reprimiera ${ }^{11}$. Probablemente nacimos admirando la naturaleza en la que nacimos; nuestra primera experiencia estética de la naturaleza debió de ser la de la sabana africana en la que nos desarrollamos como especie. Algunos se preguntan incluso si ese primer paisaje que nos maravilló no habría inspirado la descripción del jardín del Edén, o si no sería esa imagen recordada de la sabana, de extensiones de hierba salpicadas de árboles y recorridas por riachuelos, con elevaciones y refugios, lo que continuamos intentando evocar en una gran mayoría de los jardines que creamos. Que todavía conservemos esa imagen del primer paisaje contemplado en nuestra memoria de especie, y que incluso nuestros gustos estéticos en materia de paisaje puedan estar influidos por ella, es algo que no somos capaces de demostrar. Pero si tenemos esa memoria, más nos vale conservarla. Dado el camino que ha tomado la humanidad, es bien posible que dentro de algunos siglos, lo único que nos quede de ese primer paisaje que vio al nacer la humanidad, y de tantos otros que descubrimos después en la historia de una gran aventura de conquista, sea, precisamente, la memoria.

\section{NOTAS}

\footnotetext{
1 Un compendio de las diferentes opciones puede encontrarse en M. Tafalla (ed.), Los derechos de los animales, Barcelona, Idea Books, 2004.

2 Una exposición sistemática de la diversidad de corrientes puede encontrarse en Andrew Light y Holmes Rolston III (eds.), Environmental Ethics. An Anthology, Blackwell, 2003.

${ }^{3}$ Citado en Andrew Light y Holmes Rolston III (eds.), Environmental Ethics. An Anthology, ed. cit., p. 25.
}

4 Jürgen Habermas, Aclaraciones a la ética del discurso, Madrid, Trotta, 2000, p. 231 (las cursivas son mías).

${ }^{5}$ Baird Callicott, Companion to a Sand County Almanac, University of Wisconsin Press, 1988, p. 158 (las cursivas son mías)

6 Publicado en B. Williams y A. Montefiore (eds.) British Analytical Philosophy, Londres, Routledge, 1966. 
${ }^{7}$ Frankfurt Suhrkamp, 1970. Traducida en Akal, Madrid, 2004.

8 T. W. Adomo, Teoría Estética, Madrid, Akal, 2004, p. 88

9 M. Tafalla, Theodor W. Adorno. Una filosofia de la memoria, Barcelona, Herder, 2003.

10 Véase, entre otros, M. Seel, Eine Ästhetik der Natur, Frankfurt, Suhrkamp, 1996; A. Berleant A. Carlson (eds.), Journal of Aesthetics and Art Criticism. Special Issue: Environmental Aesthetics, 56:2,
1998; G. Böhme, Für eine ökologische Naturästhetik, Frankfurt, Suhrkamp, 1999; A. Carlson, Aesthetics and the Environment, Londres, Routledge, 2000; E. Brady, Aesthetics of the Natural Environment, The University of Alabama Press, 2003; A. Carlson y A. Berleant (eds.), The Aesthetics of Natural Environments, Ontario, Broadview Press, 2004.

11 Véase, entre otros, E. O. Wilson, Biophilia, Harvard University Press, 1986; E. O. Wilson (ed.), The Biophilia Hypothesis, Island Press, 1995. 